DE

M E D I C I N A

T R O P I C A L

$\mathrm{DE}$

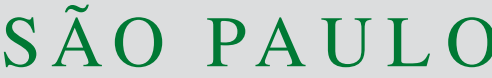

JOURNAL OF THE SÃO PAULO INSTITUTE OF TROPICAL MEDICINE

${ }^{1}$ Mohammed VI University Hospital, Department of Infectious Diseases, Marrakesh, Morocco

${ }^{2}$ Cadi Ayyad University, Marrakesh, Morocco

${ }^{3}$ Mohammed VI University Hospital, Microbiology Laboratory, Marrakesh, Morocco

Correspondence to: Zahid Talibi Alaoui Mohammed VI University Hospital, Department of Infectious Diseases, Avenue Ibn Sina Amerchich, 40000, Marrakech, Morocco

Tel: +212 0524300700

E-mail: zahidtalibi@gmail.com

Received: 6 May 2021

Accepted: 12 July 2021

\section{The first description of liver abscesses due to Salmonella enterica subsp. enterica in an African HIV-infected young woman: case report and review of the literature}

\author{
Zahid Talibi Alaoui $^{\circledR 1,2}$, Fatima Arabi ${ }^{1,2}$, Fatima Ihbibane ${ }^{1,2}$, Nabila Soraa $^{2,3}$, \\ Noura Tassi $i^{1,2}$
}

\section{ABSTRACT}

Salmonella is Gram-negative bacilli that cause a foodborne infections. When the disease occurs in patients living with HIV (PLWHA), salmonellosis is an AIDS defining illness. Here we describe the case of a 26-year-old HIV-infected female patient who was hospitalized for pain in the right upper quadrant of the abdomen, and whose explorations revealed a liver abscess due to salmonella enterica enterica that progressed well after needle aspiration and antibiotic treatment.

KEYWORDS: Salmonella. AIDS. Liver abscess. HIV. Bacteriology.

\section{INTRODUCTION}

Salmonella spp are Gram-negative bacilli, characterized by $\mathrm{O}, \mathrm{H}, \mathrm{Vi}$ antigens, and flagellum. It is a foodborne infection ${ }^{1}$ with signs and symptoms varying from gastroenteritis to enteric fevers, and focal abscesses are rare ${ }^{1}$. When it occurs in patients living with HIV (PLWHA), salmonellosis is an AIDS defining illness ${ }^{2}$. We present the case of bacteriologically-confirmed liver abscess due to Salmonella in a young PLWHA woman.

\section{CASE REPORT}

Here we describe the case of a 26-year-old female patient with an HIV-1 infection (PLWHA) initially revealed by a pulmonary tuberculosis correctly treated and cured, who was on a combined antiviral triple therapy based on efavirenz/ emtricitabine/tenofovir disoproxil (TDF/FTC/EFV) for three years, but which she stopped one year before the present hospitalization. She sought the emergency department for a one month complaint of melena with chronic liquid diarrhea at a rate of three stools a day, accompanied by fever and weight loss, but without rectal syndrome. On admission, the patient was conscious, presented with a Glasgow coma score of $15 / 15$, was hemodynamically and respiratory stable, febrile with a body temperature of $38^{\circ} \mathrm{C}$, heart rate of 100 per min, respiratory rate of 20 cycles per min, blood pressure of $110 / 40 \mathrm{mmHg}$, weighting $46 \mathrm{~kg}$, in addition to an oral candidiasis with possible esophageal involvement. On the abdominal examination, the patient had pain on the right upper quadrant, epigastric tenderness associated with a homogeneous hepatomegaly $(21 \mathrm{~cm})$ and splenomegaly $(14 \mathrm{~cm})$ and the hernia orifices were free. The abdominal ultrasound showed multiple collections at the level of the segments I, IV, and V related to liver abscesses of which the 
largest lesion measured 5.8 by $4.2 \mathrm{~cm}$ with hepatomegaly associated with an anechoic peritoneal effusion of moderate abundance (Figure 1). The patient was treated empirically with ceftriaxone, metronidazole and gentamicin for the liver abscesses, fluconazole for oral and esophageal candidiasis, and a medication to slow down the intestinal transit.

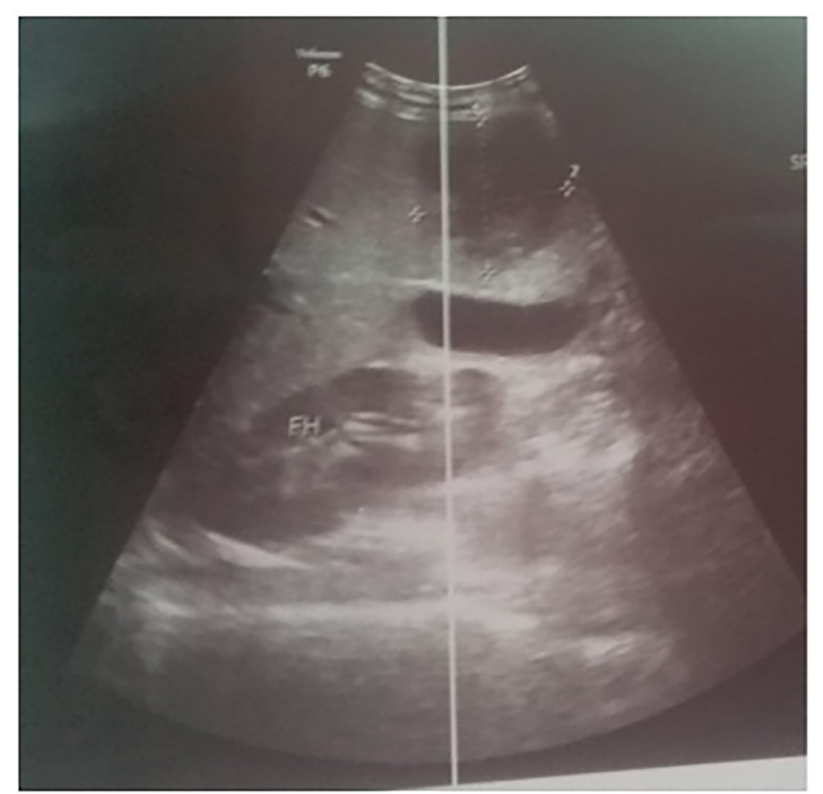

Figure 1 - Ultrasound of the liver showing an abscess due to Salmonella sp. at the level of the segment IV associated with hepatomegaly: $1=5.8 \mathrm{~cm} ; 2=4.2 \mathrm{~cm}$; $\mathrm{FH}=$ size of hepatomegaly.

On the blood count, the patient had normochromic normocytic anemia, $5.8 \mathrm{~g} / \mathrm{dL}$ of hemoglobin $(11.5-16.5)$, lymphopenia, with $840 / \mathrm{mm}^{3}(1,000-4,000)$, normal leukocytes - $6,040 / \mathrm{mm}^{3},(3,600-11,000)$, normal neutrophils $-4,490 / \mathrm{mm}^{3}(1,800-7,500)$, and a normal platelet count of $166,000 / \mathrm{mm}^{3},(140,000-400,000)$, and the CD4 - positive T cell count was 27 cells $/ \mathrm{mm}^{3}$. She had hepatitis flares and cholestasis with AST $162 \mathrm{IU} / \mathrm{L}$ $(5 \mathrm{~N})(<30)$, ALT $55 \mathrm{IU} / \mathrm{L}(\mathrm{N})(<50)$, ALP $495 \mathrm{IU} / \mathrm{L}(5 \mathrm{~N})$ (30-100), $\gamma$ GT $280 \mathrm{IU} / \mathrm{L}(6 \mathrm{~N})(<45)$, CRP $117 \mathrm{mg} / \mathrm{L}$ $(<5 \mathrm{mg} / \mathrm{L})$, normal renal function; negative serology to hydatid, amebiasis, hepatitis B virus (HBV), hepatitis C virus ( $\mathrm{HCV}$ ), syphilis and toxoplasmosis, while serology to CMV IgG was positive but negative to IgM, COVID-19 RT-PCR and IgG antibodies raised to SARS-CoV-2 were negative, and the patient also presented with negative sputum evaluated by the Xpert MTB/RIF (Genexpert ${ }^{\circledR}$ Cepheid Inc, California, USA) and three Acid-Fast Bacilli (AFB) smear microscopy, three cultures of stool and parasitological stool examination were also negative, in addition to a blood culture.

An ultrasound-guided puncture was performed which revealed the presence of a Salmonella enterica subsp. enterica in the purulent secretion that was only resistant to cefalotin, cefoxitin, gentamicin, amikacin, but was sensitive to amoxicilline (Figure 2). The Genexpert ${ }^{\circledR}$ on the purulent secretion was negative. A wet smear was negative for Entamoeba histolytica trophozoite. Next, we stopped metronidazole and gentamicin and we continued the treatment with intravenous amoxicillin plus clavulanic acid $1 \mathrm{~g}$ tid for eight days; then we switched to oral amoxicillin $1 \mathrm{~g}$ tid during three weeks and the antiretroviral therapy was restarted. Ten days after the treatment, the patient improved clinically and the CRP decreased to $13 \mathrm{mg} / \mathrm{L}$. A follow-up ultrasound showed that the size of the abscess had decreased and the patient had a favorable outcome.

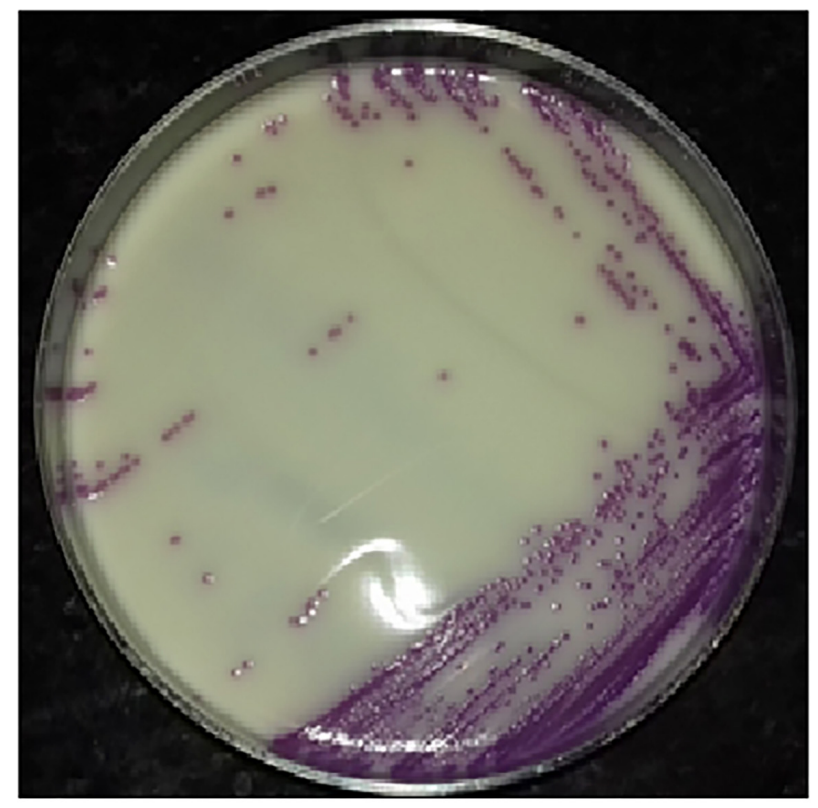

Figure 2 - Morphology of Salmonella enterica subsp. enterica colonies on the Chromogene OXOID ${ }^{\mathrm{TM}}$ Brilliance $^{\mathrm{TM}}$ medium.

\section{DISCUSSION}

Salmonellosis is a major public health problem in resource-constraint countries ${ }^{1}$. Severe forms of Salmonella infection usually occur in immunocompromised hosts ${ }^{1}$. To our knowledge this case is the first description of an African PLWHA with bacteriologically-confirmed liver abscess caused by Salmonella and the third case in the world ${ }^{2,3}$.

The pathogens responsible for liver pyogenic abscesses are Streptococci, Staphylococci, Escherichia coli, Klebsiella pneumonia, bacteroides and Enterococci ${ }^{4}$. Isolation of Salmonella from a liver abscess is rare, and its occurrence in HIV-infected individuals is very exceptional. A review of the literature on the PubMed database using the terms: 
Table 1 - Review of the literature on Salmonella spp. liver abscess cases in HIV-infected patients and recent published cases in non-HIV patients.

\begin{tabular}{|c|c|c|c|c|c|c|c|c|c|c|c|c|c|c|}
\hline \multirow{2}{*}{$\begin{array}{l}\text { Publication } \\
\text { year }\end{array}$} & \multirow{2}{*}{$\begin{array}{c}\text { Type of } \\
\text { HIV }\end{array}$} & \multirow{2}{*}{$\begin{array}{l}\text { CD4 count } \\
\text { (cells } / \mathrm{mm}^{3} \text { ) }\end{array}$} & \multirow{2}{*}{$\begin{array}{c}\text { Age } \\
\text { (years) }\end{array}$} & \multirow{2}{*}{ Sex } & \multirow{2}{*}{$\begin{array}{l}\text { Unique or } \\
\text { multiple }\end{array}$} & \multicolumn{4}{|c|}{ Isolated from } & \multicolumn{3}{|c|}{ Treatment } & \multirow{2}{*}{ Outcome } & \multirow{2}{*}{ Reference } \\
\hline & & & & & & Abscess & Blood & Stool & Other & Antibiotics & Drainage & Surgery & & \\
\hline 2017 & - & - & 27 & - & Unique & $x$ & $x$ & & & $x$ & $x$ & $x$ & Good & Ladic et al. ${ }^{9}$ \\
\hline 2017 & - & - & 53 & $\mathrm{~F}$ & Unique & $x$ & & & & $x$ & $x$ & $x$ & Good & Tee Yu et al. ${ }^{12}$ \\
\hline 2015 & - & - & 65 & M & Multiple & $x$ & $x$ & & & $x$ & $x$ & & Good & $\begin{array}{c}\text { Kamatani } \\
\text { et al. }{ }^{17}\end{array}$ \\
\hline 2015 & - & - & 64 & M & Multiple & & & & $\times$ Ascites & $x$ & & & Dead & Hung et al. ${ }^{13}$ \\
\hline 2014 & - & - & 30 & $\mathrm{M}$ & Unique & $x$ & & & $\begin{array}{l}\times \text { Pleura } \\
\text { fluid }\end{array}$ & $x$ & $x$ & & Dead & Mahajan et al. ${ }^{11}$ \\
\hline 2014 & - & - & 54 & M & Unique & $x$ & & & & $x$ & $x$ & & Dead & Imoto et al. ${ }^{8}$ \\
\hline 2014 & - & - & 24 & M & Multiple & $x$ & $x$ & & & $x$ & $x$ & & $\begin{array}{l}\text { Lost to } \\
\text { follow-up }\end{array}$ & Jorge et al. ${ }^{14}$ \\
\hline 2013 & - & - & 58 & M & Unique & $x$ & $x$ & & & $x$ & $x$ & $x$ & Good & Qu et al..$^{10}$ \\
\hline 2013 & - & - & 43 & M & Unique & & & $x$ & & $x$ & & & Good & Tulachan et al. ${ }^{15}$ \\
\hline 2011 & _- & - & 56 & M & Unique & $x$ & & & & $x$ & $x$ & & Good & Sheikh et al. ${ }^{6}$ \\
\hline 2006 & - & - & 8 & $M$ & Multiple & & $x$ & & & $x$ & $x$ & & Good & $\begin{array}{l}\text { Kabra and } \\
\text { Wadhwa }^{16}\end{array}$ \\
\hline 2019 & HIV-1 & 88 & 51 & M & Unique & $x$ & & & & $x$ & $x$ & & Good & Lin et al. ${ }^{3}$ \\
\hline 2003 & HIV-1 & 265 & 31 & M & Unique & $x$ & & $x$ & & $x$ & $x$ & & Good & Vidal et al. ${ }^{2}$ \\
\hline 2021 & HIV-1 & 27 & 26 & $\mathrm{~F}$ & Multiple & $x$ & & & & $x$ & $x$ & & Good & This study \\
\hline
\end{tabular}

salmonella, salmonellosis, liver abscess, hepatic abscess, in the period from 1952 to 2021 found only 26 cases of which only two occurred in PLWHA ${ }^{2,4-17}$ (Table 1).

The weight loss was constant in our patient and in the two other published cases of liver abscesses due to Salmonella in PLWHAs ${ }^{2,3}$. The mechanism behind this is the chronic diarrhea leading to severe malnutrition and dehydration. Ultrasound images of the liver Salmonella abscess like the amebic abscess shows a solitary hypoechoic image located in the middle of the liver or in the right liver, unlike other pyogenic abscesses that most often manifest by multiple hypoechoic images. Imaging results obtained by ultrasound or CT scan can guide the liver puncture so as to isolate the causative pathogen².

For uncomplicated Salmonella gastroenteritis, the antibiotic treatment is not recommended because it does not reduce the duration of the disease, on the contrary, it significantly prolongs the duration of the fecal excretion of the bacteria and increases the risk of resistance to antibiotics ${ }^{1}$. Salmonellosis should only be treated with antibiotics when there is bacteremia, enteric fever, focal infection or abscesses.

The treatment of abscesses can be limited to the antibiotic therapy if the size of the abscess is less than $4 \mathrm{~cm}^{2,15}$. The combination of needle aspiration and antimicrobial therapy for treating multiple pyogenic liver abscesses is associated with a similar efficacy but a shorter hospitalization time compared to drainage of the abscess only. The percutaneous drainage is indicated for patients who continue to be febrile even after $72 \mathrm{~h}$ of adequate medical treatment and aspiration; liver abscesses $>6 \mathrm{~cm}$; and clinical or ultrasound features suggesting impending perforation, but drainage should always be undertaken before surgery to promote the comfort of the patient and reduce costs. The surgical drainage is reserved for patients who do not respond to treatment with percutaneous aspiration or drainage and antibiotics or who have concomitant intra-abdominal disease requiring a surgical intervention ${ }^{18}$. Our patient had multiple liver abscesses and improved well after needle aspiration associated with antibiotic treatment.

\section{CONCLUSION}

We conclude that even though it is rare, a liver abscess due to Salmonella in a PLWHA is a diagnosis that should not be overlooked among the pyogenic causes of liver abscesses and that a careful guided needle aspiration together with an antibiotic treatment is frequently associated with a good prognosis.

\section{CONFLICT OF INTERESTS}

There are no financial or other relationships of any kind of the authors that might lead to any conflict of interests. 


\section{FUNDING}

There are no sources of funding or study sponsors that have supported the work.

\section{REFERENCES}

1. Giannella RA. Salmonella. In: Baron S, editor. Medical microbiology. $4^{\text {th }}$ ed. Galveston: University of Texas Medical Branch at Galveston; 1996. [cited 2021 Jul 15]. Available from: http://www.ncbi.nlm.nih.gov/books/NBK8435/

2. Vidal JE, Silva PR, Schiavon Nogueira R, Bonasser Filho F, Hernandez AV. Liver abscess due to Salmonella enteritidis in a returned traveler with HIV infection: case report and review of the literature. Rev Inst Med Trop Sao Paulo. 2003;45:115-7.

3. Lin P, Ko WC, Lin WC, Li MC. Liver abscess caused by coexisting Salmonella enteritidis and Entamoeba histolytica in a HIVinfected patient. J Microbiol Immunol Infect. 2019;52:836-7.

4. Poon MC, Sanders MG. Hepatic abscess caused by Salmonella paratyphi B. Can Med Assoc J. 1972;107:529-31.

5. Rovito V, Bonanno CA. Salmonella hepatic abscess: an unusual complication of the Salmonella carrier state? Am J Gastroenterol. 1982;77:338-9.

6. Sheikh I, Sievers C, Mullen K. Salmonella enteritidis liver abscess. Ann Hepatol. 2011;10:370-1.

7. Hirschowitz BI. Pyogenic liver abscess: a review with a case report of a solitary abscess caused by Salmonella enteritidis. Gastroenterology. 1952;21:291-9.

8. Imoto A, Ooi Y, Edogawa S, Ogura T, Masuda D, Mohamed $\mathrm{M}$, et al. Liver abscess caused by CTX-M-55-type extendedspectrum $\beta$-lactamase (ESBL)-producing Salmonella enteritidis. Intern Med. 2014;53:1699-703.
9. Ladic A, Petrovic I, Gojevic A, Kinda E, Coza I. Salmonella enterica serovar enteritidis liver abscess after blunt abdominal trauma. Balkan Med J. 2017;34:469-72.

10. Qu F, Fan Z, Cui E, Zhang W, Bao C, Chen S, et al. First report of liver abscess caused by Salmonella enterica serovar Dublin. J Clin Microbiol. 2013;51:3140-2.

11. Mahajan RK, Sharma S, Madan P, Duggal N. Pyogenic liver abscess caused by Salmonella Enteritidis: a rare case report. Indian J Pathol Microbiol. 2014;57:632-4.

12. Tee Yu HH, Tsang S, Cheung TT. An unusual cause of acute abdomen-gas-forming liver abscess due to Salmonella enteritidis. Asian J Surg. 2017;40:66-9.

13. Hung YL, Wang CC. Hepatobiliary and pancreatic: gas-forming liver abscess caused by Salmonella after transcatheter arterial chemoembolization for hepatocellular carcinoma. J Gastroenterol Hepatol. 2015;30:1449.

14. Jorge JF, Costa AB, Rodrigues JL, Girão ES, Luiz RS, Sousa AQ, et al. Salmonella typhi liver abscess overlying a metastatic melanoma. Am J Trop Med Hyg. 2014;90:716-8.

15. Tulachan SS, Wilkins CE, Cutrona AF, Gemmel D, Marnejon TP. Hepatic abscess associated with Salmonella serotype B in a chronic alcoholic patient. Hepatobiliary Pancreat Dis Int. 2013;12:440-2.

16. Kabra S, Wadhwa V. Hepatic abscess caused by Salmonella typhi. Indian Pediatr. 2006;43:81-2.

17. Kamatani T, Okada T, Iguchi H, Takahashi Y, Yokomori H. Liver abscess caused by Salmonella choleraesuis. Int J Gen Med. 2015;8:155-61.

18. Lübbert C, Wiegand J, Karlas T. Therapy of liver abscesses. Viszeralmedizin. 2014;30:334-41 